\title{
Rigid Stabilization of Partial Incongruous Lisfranc Dislocations: A Cannulated Solid Screw Technique
}

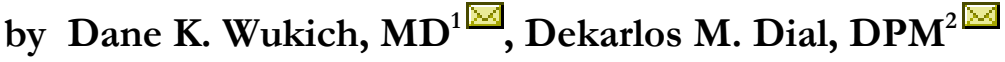

The Foot and Ankle Online Journal 2 (6): 4

For several years, there has been much controversy over the optimal form of fixation in the operative treatment of Lisfranc injuries. Both cortical and cannulated screws have been widely used in the treatment of these injuries. The level of technical difficulty and reproducible accuracy of the cannulated screw system has gained much popularity. In comparison, the rigid stability of cortical screws appears more favorable. The authors present a cannulated technique utilizing a single $4.0 \mathrm{~mm}$ cortical screw (Synthes USA Paoli, Pa.). In Lisfranc injuries with partial incongruity, this method allows precise screw placement while maintaining rigid solid screw stabilization. The technique is minimally invasive, provides anatomical restoration and allows early return to functional activity.

Key Words: Lisfranc injury, midfoot fracture, cannulated screw, foot sprain, dislocation

Accepted: May, 2009

Published: June, 2009

This is an Open Access article distributed under the terms of the Creative Commons Attribution License. It permits unrestricted use, distribution, and reproduction in any medium, provided the original work is properly cited. @The Foot and Ankle Online Journal (www.faoj.org)

L isfranc fracture dislocations account for $0.2 \%$ of all fractures. ${ }^{1,2}$ In 1909, Quenu and Kuss described the first classification system for Lisfranc injuries. ${ }^{3}$ This classification system was modified by Hardcastle in 1982. In 1986, Myerson, et al., ${ }^{5}$ further modified the Hardcastle classification into medial and lateral dislocations. Type A is a total incongruent tarsometatarsal joint complex. Types B1 is a partial incongruity with medical displacement affecting the first ray or first metatarsal, and B2 is partial incongruity resulting in lateral displacement of one or more lesser metatarsals. The most common Lisfranc injury in the athlete is the partial incongruous injury resulting in medial or lateral dislocation. ${ }^{4,17,18,19}$

\footnotetext{
Address correspondence to: Dekarlos M. Dial, DPM, Cornerstone Foot and Ankle Specialists, 1814 West Chester Drive, Suite 300, High Point, North Carolina 27262

${ }^{1}$ Chief, Foot and Ankle Division, Department of Orthopaedic Surgery; University of Pittsburgh Medical Center, Pittsburgh, Pennsylvania.

${ }^{2}$ 3rd year resident, Department of Graduate Medical Education; University of Pittsburgh Medical Center Surgery, Pittsburgh, Pennsylvania.
}

Types C1 and C2 injuries result in partial or total displacement respectfully. Such classification systems allow communication between surgeons, but offer no prognostic value.

Stable anatomic reduction and internal fixation for Lisfranc fracture dislocations has been described using both screw and Kirchner wire fixation. Pin migration, infection and loss of reduction have been reported with Kirchner wire fixation. ${ }^{11}$ Over the years, screw fixation has emerged as the superior fixation method for stabilizing the medial three tarsometatarsal joints. ${ }^{1,6,7,9,10}$ Some disadvantages of screw fixation include articular cartilage damage and screw breakage. ${ }^{12}$

The authors share their surgical technique for addressing partial incongruous Lisfranc injuries; lateral displacement injuries affecting only the second metatarsal. 


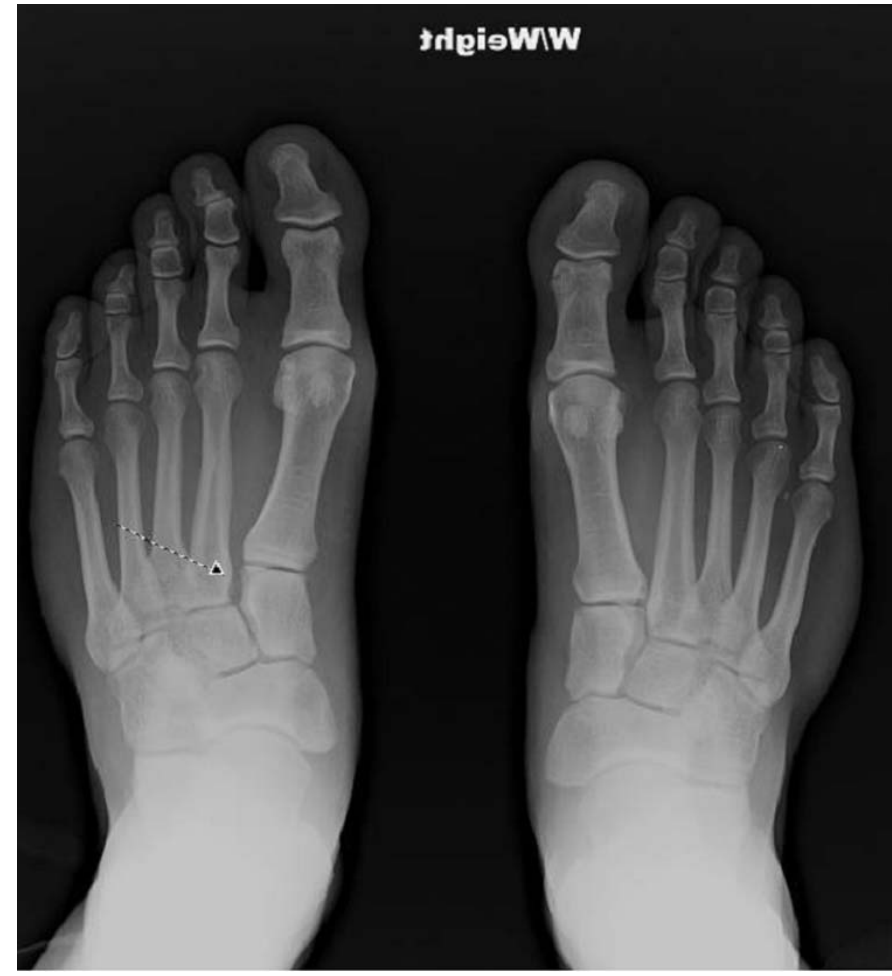

Figure 1 Bilateral comparison weightbearing anteroposterior radiographs demonstrating partial incongruity of the second tarsometatarsal joint. Note the arrow identifying the first and second metatarsal base diastasis.

The technique is also useful for patients with a symptomatic subtle diastasis. This technique allows direct visualization of the injury, anatomical reduction, articular cartilage preservation, and rigid solid screw stabilization. The technique is avoided in patients with neuropathic arthropathy, peripheral vascular disease, insensate feet, open physis, complex dislocations, and open fracture dislocations.

\section{Surgical Technique}

Patients with partially incongruent Lisfranc injuries (B2) were treated with internal fixation utilizing 4.0 mm solid screw (Synthes ${ }^{\circledR}$, Paoli, Pa. USA).

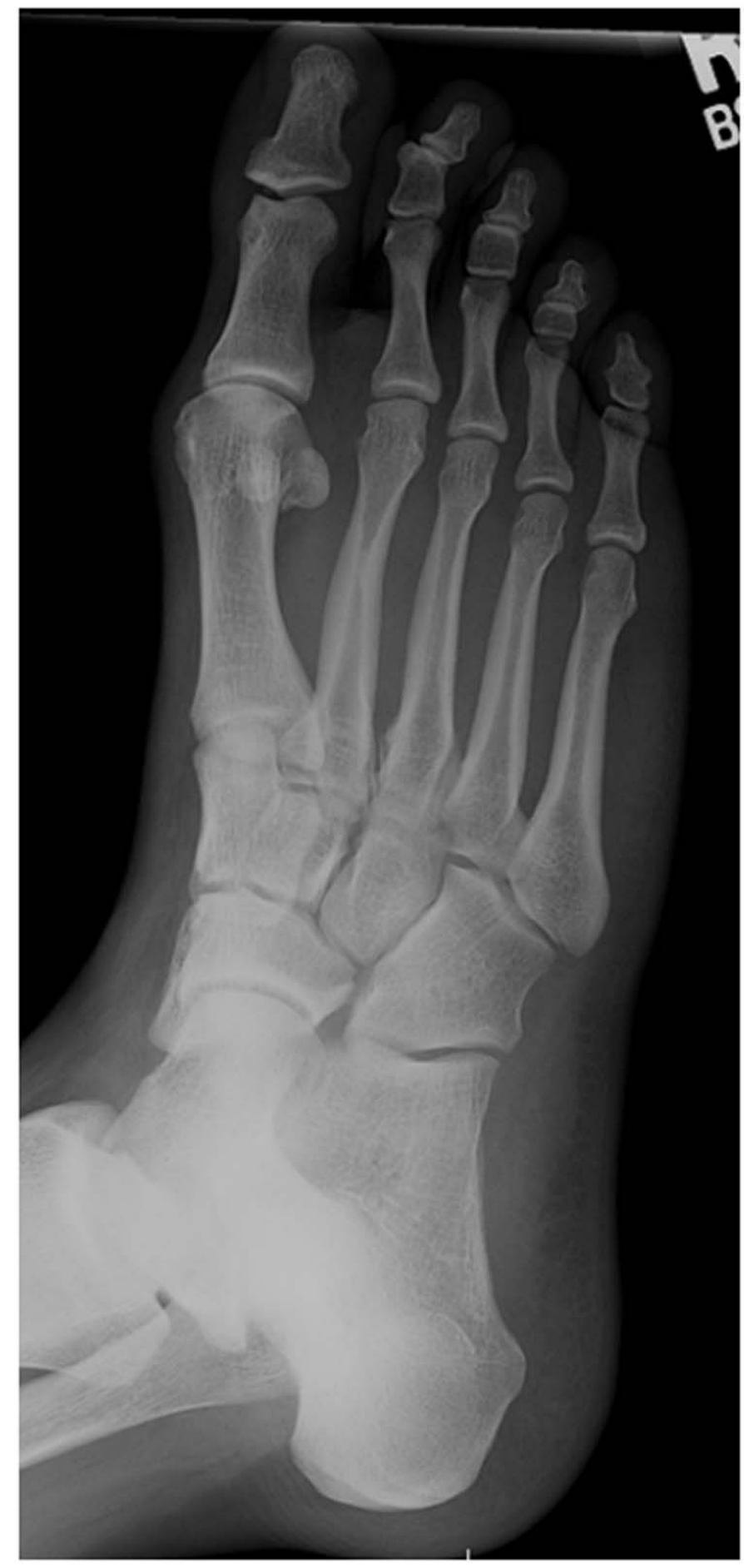

Figure 2 Oblique foot radiograph. Note the collinear relationship at the adjacent tarsometatarsal joints. 


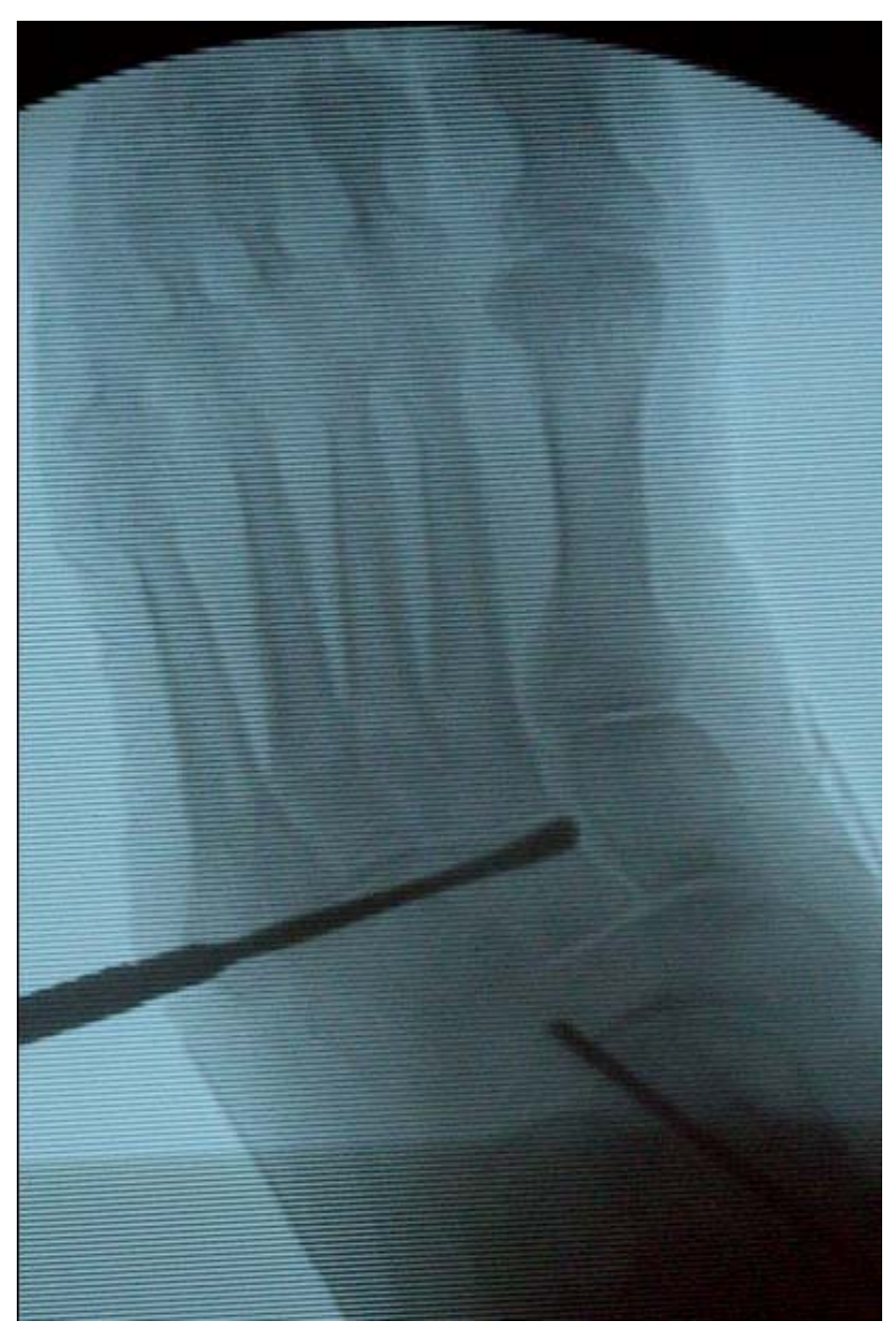

Figure 3 Freer elevator used prior to guide incision placement to identify the interval between the first and second metatarsal cuneiforms.

Pre-operatively, all patients receive a regional popliteal and femoral block. With the patient lying supine, a stress abduction stress is applied to the midfoot as described by Coss, et al. ${ }^{13}$ Stress radiography may obviate the need for stabilization of the entire tarsometatarsal joint complex. (Figs. 1 and 2)

The neurovascular bundle is identified by palpating the dorsalis pedis artery prior to inflation of the calf tourniquet. The interval between the first and second metatarsal bases is verified under fluoroscopy. (Fig. 3) A single $6 \mathrm{~cm}$ longitudinal skin incision is made medial to the neurovascular bundle.

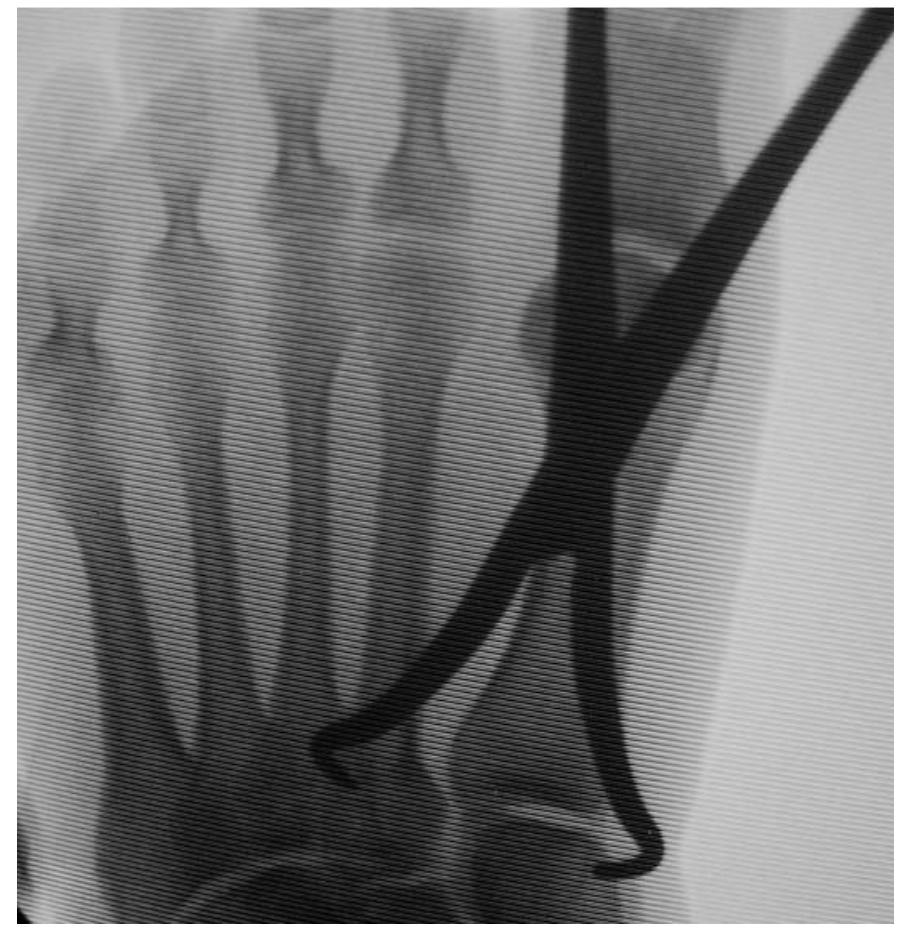

Figure $4 \mathrm{~A}$ bone tenaculum is utilized to reduce the first and second metatarsal base diastasis.

Blunt dissection is continued down to the level of the extensor hallucis brevis muscle tendon (EHB). The EHB tendon is retracted laterally.

Subperiosteal dissection at the intercuneiform level is extended just distal to the first and second metatarsal base articulation. The Lisfranc ligament integrity is evaluated. A ruptured Lisfranc ligament, fibrotic tissue and or bone fragments can impede anatomical reduction and must be debrided and or excised. Care is taken to protect the neurovascular bundle and deep plantar artery.

Reduction is performed by placing a bone tenaculum around the medial cuneiform and second metatarsal base. The second metatarsal is reduced to the medial border of the middle cuneiform. The reduction is verified under fluoroscopy. (Fig. 4) If a diastasis remains present, first and second metatarsal base interval is inspected and the bone reduction tenaculum is adjusted. Once reduction of the diastasis is achieved, a $1.2 \mathrm{~mm}$ guide wire is obtained from the cannulated 3.5/4.0mm Synthes screw set. 


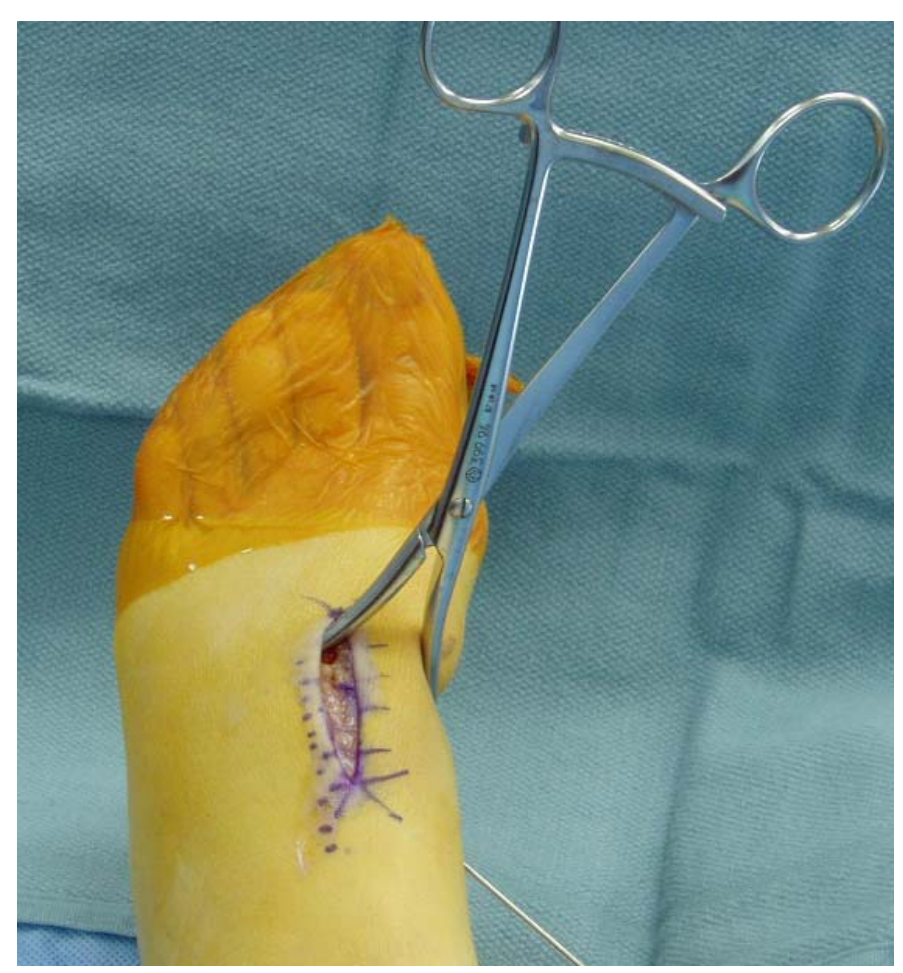

Figure 5 The $1.2 \mathrm{~mm}$ guide wire is placed from medial to lateral just penetrating the second metatarsal base lateral cortex.

The $1.2 \mathrm{~mm}$ guide wire is placed obliquely from the medial cuneiform (plantar proximal) into the second metatarsal base (distal dorsal) penetrating the lateral cortex. (Fig. 5) The knee is then flexed and the guide wire position is verified under fluoroscopy on both an anteroposterior and lateral projection. The medial cuneiform is then countersunk to prevent screw irritation. Next, the cannulated depth gauge is used to determine the screw length. The $1.2 \mathrm{~mm}$ guide wire is advanced until it exits the foot dorsolaterally. (Fig. 6A and $6 \mathrm{~B})$. By exiting the foot in this fashion, it allows easy retrieval of the guide wire if breakage occurs. The cannulated $2.5 \mathrm{~mm}$ drill bit is utilized to drill over the guide wire and penetrates the lateral second metatarsal base cortex. (Fig. 7) The appropriate length $4.0 \mathrm{~mm}$ screw is obtained and inserted in line with the guide wire. (Fig. 8A and 8B) To prevent toggle or misdirection during insertion, the guide wire is removed simultaneously.

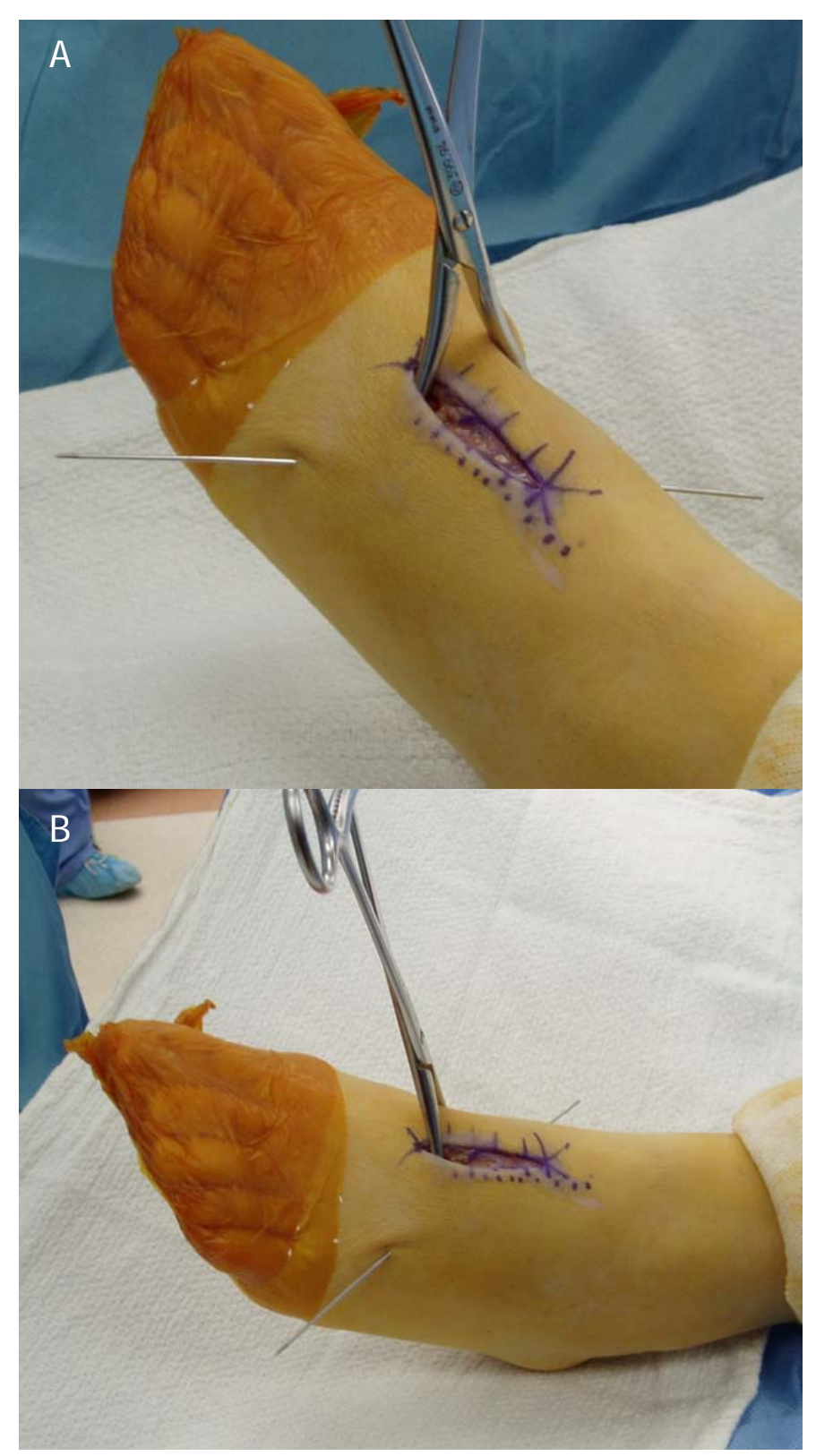

Figures 6A and 6B The $1.2 \mathrm{~mm}$ guide wire is advanced obliquely until exiting the foot laterally allowing retrieval if breakage occurs. (A) Note the direction of the guide wire in both the transverse and sagittal planes. (B)

The bone reduction tenaculum is disengaged and removed. Screw placement and stability are verified under fluoroscopy. (Fig. 9) The calf tourniquet is deflated and hemostasis achieved prior to closure. 


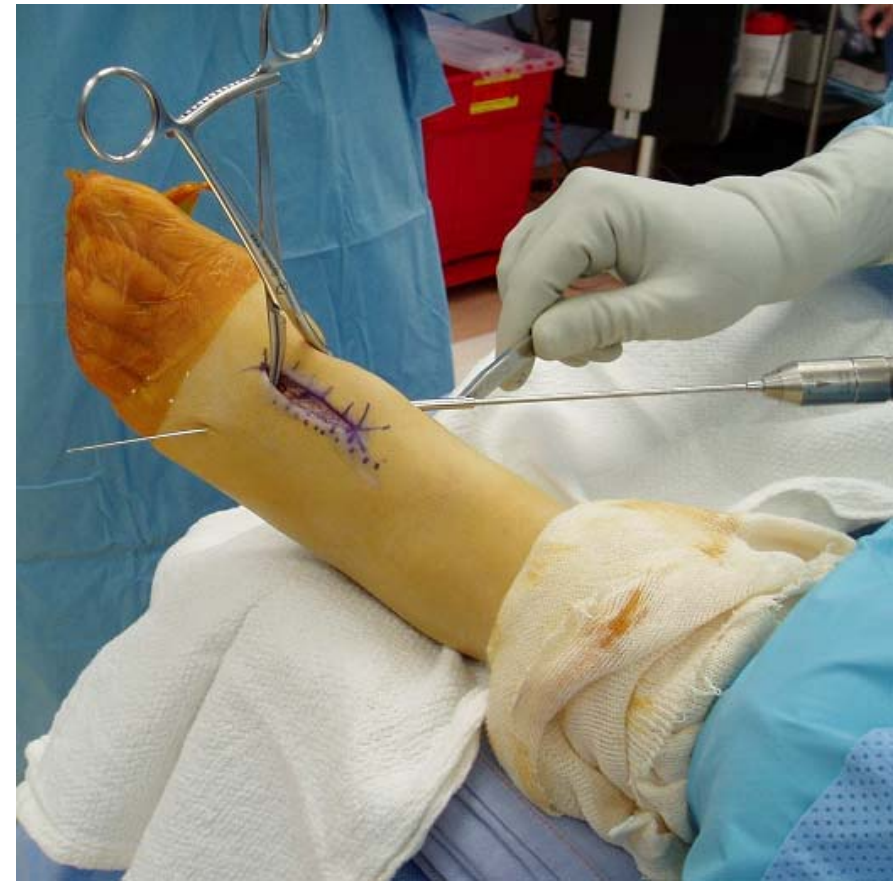

Figure 7 The $2.5 \mathrm{~mm}$ cannulated drill is used to drill over the $1.2 \mathrm{~mm}$ guide wire penetrating the lateral $2 \mathrm{nd}$ metatarsal base cortex.

Postoperatively, patients are placed into a Jones compression dressing with a posterior splint for until postoperative day seven. The patient is then transferred into a short leg non-weightbearing cast for 14 days. At day 21 the sutures are removed and another short leg cast is applied. Patients are nonweightbearing for a further 6 weeks, followed by protected weight bearing in a walking boot with progression to normal shoe gear as tolerated.

\section{Discussion}

The standard treatment for Lisfranc joint injuries is to achieve anatomic reduction with internal fixation. Stable anatomical reduction results in more favorable long-term outcomes. ${ }^{4,714}$ Buzzard, et al., reported that optimal results are obtained if precise anatomical reduction is achieved. ${ }^{1}$ Kuo, et al., reported that stable anatomic reduction lead to better long-term outcomes with higher AOFAS midfoot scores. ${ }^{7}$

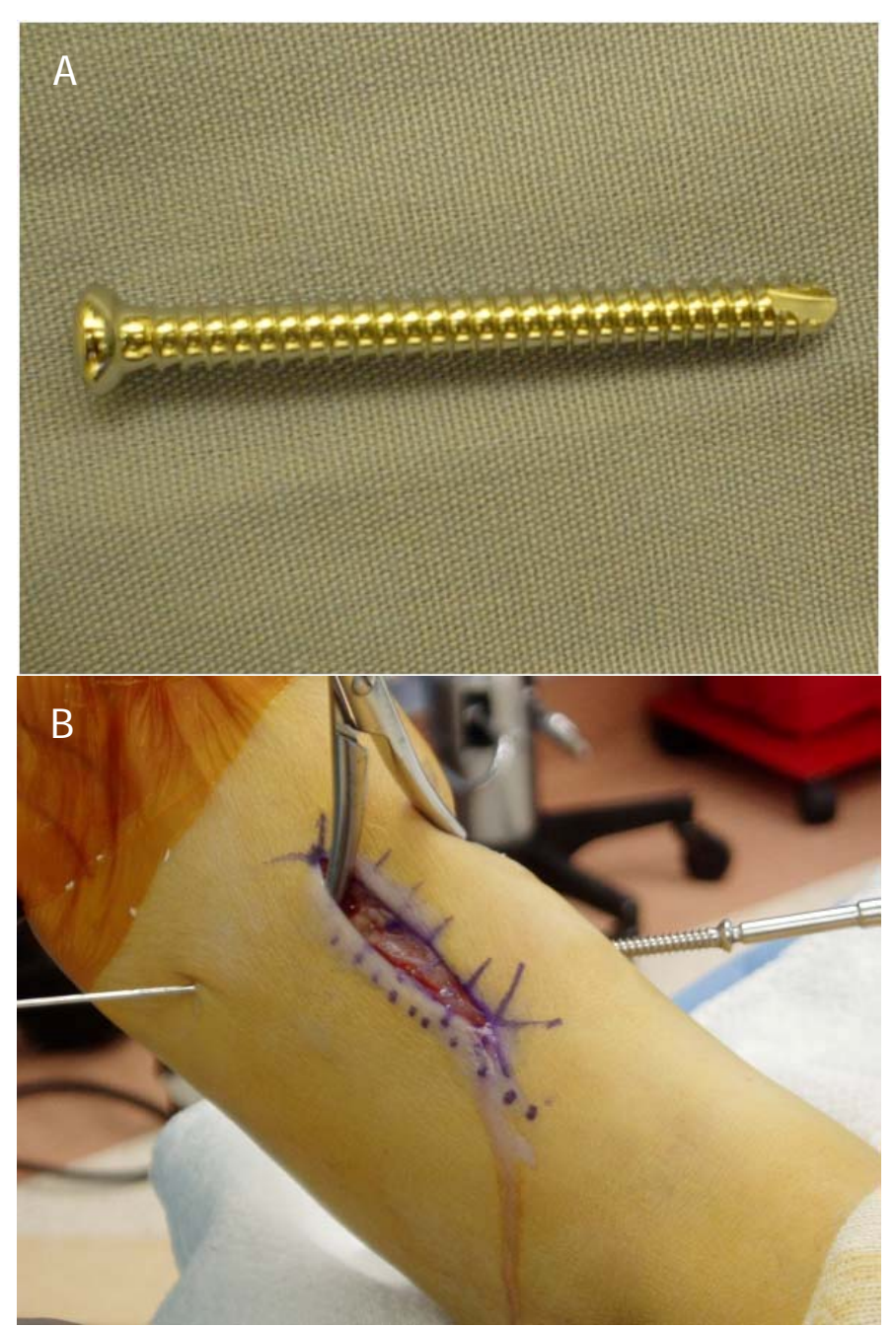

Figures 8A and 8B $4.0 \mathrm{~mm}$ cortical screw (Synthes ${ }^{\circledR}$ Paoli, Pa. USA) (A) Simultaneous placement of the $4.0 \mathrm{~mm}$ solid screw and removal of the $1.2 \mathrm{~mm}$ guide wire. (B)

Current recommendations support screw fixation in treatment of Lisfranc injuries. However, screw fixation is not without complications. Some authors believe that screw fixation results in articular cartilage damage during and that there is added risk for screw breakage. ${ }^{15}$ Our technique preserves cartilage congruity and provides stabilization until ligamentous healing is restored. Thodarson, et al., found that absorbable screw fixation (PLA screws) was safe and eliminated the need for removal. ${ }^{5}$ 


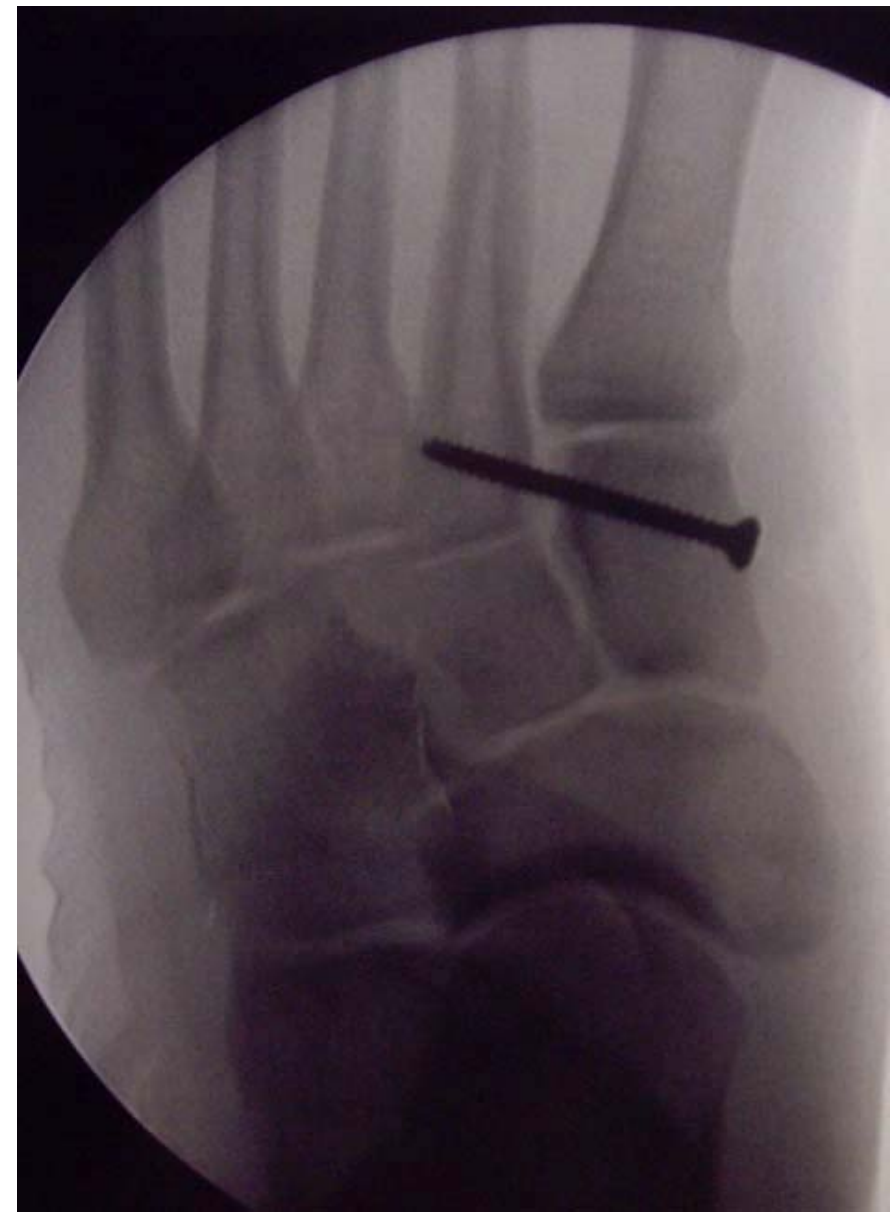

Figure 9 Intraoperative anteroposterior image demonstrating diastasis reduction and $4.0 \mathrm{~mm}$ solid screw fixation.

In a cadaveric study, dorsal plating versus screw fixation showed no difference in resisting tarsometatarsal joint displacement. ${ }^{15}$

Ly, et al., recommended primary stable arthrodesis of purely ligamentous Lisfranc dislocations. The authors reviewed 41 patients with an average follow-up of 42 months. The average post-operative AOFAS score was 68.6 points in the open reduction and internal fixation group and 88 points for the arthrodesis group. The authors believe that stable arthrodesis prevented loss of correction and degenerative changes. ${ }^{16}$
The cannulated solid screw technique is an alternative treatment option for partial incongruous injuries or subtle dislocations. Excessive fixation is avoided while preserving articular congruity. The rigidity of the $4.0 \mathrm{~mm}$ solid screw allows early functional rehabilitation. Retrospectively, the authors have treated 10 patients using this technique with a zero incidence of hardware fatigue or breakage.

In conclusion, our technique has proven to be effective for stabilizing partial incongruous Lisfranc injuries. The technique is reproducible, accurate and allows for early function rehabilitation. The rigidity of the $4.0 \mathrm{~mm}$ solid screw provides optimal stability. However, care must be taken to individualize this treatment based on the extent of tarsometatarsal joint displacement.

\section{References}

1. Buzzard BM, Manos, RE, Buoncristiani A, Mills WJ: Surgical management of acute tarsometatarsal fracture dislocation in the adult. Clin Orthop 353: 125 - 133, 1998.

2. Hardcastle PH, Reschauer R, Kutscha-Lissberg E, Schoffmann W: Injuries to the tarsometatarsal joint. Incidence, classification and treatment. J Bone and Joint Surg 64B (3): 349 $-356,1982$.

3. Quenu E, Kuss G: Etude sur les luxations du metatarse (luxations metatarso-tariennes). Rev Chir 39: 281 - 336, 1909.

4. Myerson MS, Fisher RT, Burgess AR, Kenzora JE. Fracture dislocations of the tarsometatarsal joints: end results correlated with pathology and treatment. Foot Ankle Clinics 6: 225 - 242, 1986.

5. Thodarson DB, Hurwitz G: PLA screw fixation of Lisfranc Injuries. Foot Ankle Int 23: 1003 - 1007, 2002.

6. Myerson MS: The diagnosis and treatment of injury to the tarsometatarsal joint complex. J Bone Joint Surg 81B: $756-763$, 1999.

7. Kuo R, Tejwani N, DiGiovanni CW, Holt SK, Benirschke SK, Hansen ST Jr, Sangeorzan BJ: Outcome after open reduction and internal fixation of Lisfranc joint injuries. J Bone Joint Surg 82A: 1609 - 1618, 2000.

8. Arntz CT, Hansen ST: Dislocations and fracture dislocations of the tarsometatarsal joints. Orthop Clin North Am 18: 105 114, 1987.

9. Bloome DM, Clanton TO: Treatment of Lisfranc injuries in the athlete. Tech. Foot Ankle Surg 1: 94 - 101, 2002.

10. Chiodo C, Myerson M: Developments and advances in the diagnosis and treatment of injuries to the tarsometatarsal joint. Orthop Clin North Am 32: 11 - 20, 2001. 
11. Arntz CJ, Veith RG, Hansen Jr. ST: Fractures and fracture dislocations of the tarsometatarsal joints. J Bone Joint Surg 70A (2): $173-181,1988$.

12. Alberta FG, Aronow MS, Barrero M, Diaz-Doran V, Sullivan RJ, Adams DJ: Ligamentous Lisfranc joint injuries: A biomechanical comparison of dorsal plate and transarticular Screw Fixation. Foot Ankle Int 26: 462 - 473, 2005.

13. Coss HS, Manos RE, Buoncristiani A, Mills W: Abduction stress and AP weightbearing radiography of purely ligamentous injury in the tarsometatarsal joint. Foot Ankle Int 19: 537 - 541, 1998.

14. Goosens M, De Stoop N: Lisfranc's fracture-dislocations: etiology, radiology, and results of treatment. Clin Orthop 176: $165-162,1983$.

15. Alberta FG, Aronow MS, Barrero M, Diaz-Doran V, Sullivan RJ, Adams DJ: Ligamentous Lisfranc joint injuries: a biomechanical comparison of dorsal plate and transarticular screw fixation. Foot Ankle Int. 26: 462 - 472, 2005.

16. Ly V, Coetzee JC: Treatment of primary ligamentous Lisfranc joint injuries: primary arthrodesis compared with open reduction and internal fixation. J Bone Joint Surg 88A: 514 520, 2006.

17. Davies MS, Saxby TS: Intercuneiform instability and the "gap sign". Foot Ankle Int 20: 606 - 609, 1999.

18. Meyer SA, Callaghan JJ, Albright JP, Crowley ET, Powell JW: Midfoot sprains in collegiate football players. Am J Sports Medicine 22: 392-401, 1994.

19. Shapiro MS, Wascher DC, Finerman GA: Rupture of Lisfranc's ligament in athletes. Am J Sports Med 22: 687 - 691, 1994. 